\title{
Nuevos ejemplares de marcas sobre Ánforas Dressel 20 en EL TERRITORIO DEL CONVENTUS HiSPALENSIS: VILLAR TESORO, Azanaque y La Catria*
}

Nouvelles exemplaires du timbres amphoriques sur Dressel 20 dans le territoire du Conventus Hispalensis: Villar Tesoro, Azanaque et La Catria

JoRdi PÉREZ GoNZÁLEZ

CEIPAC. Universidade de Barcelona

Recibido: 18/09/2016

Aceptado: $19 / 05 / 2017$

Revisado: 21/03/2017

Publicado: 23/06/2017

\section{RESUMEN}

El presente trabajo está dedicado a profundizar entorno a las relaciones económicas y sociales establecidas entre las diversas provincias del Imperio Romano. Con el fin de conocer el rol que jugó la provincia Baetica dentro de la administración romana, y de las políticas económicas aquí desempeñadas, es necesario un mayor conocimiento de la producción alimentaria en la región. Para ello, el hallazgo de los contenedores ánforicos fabricados a lo largo del Guadalquivir y el Genil nos permite profundizar entorno a la producción y exportación de alimentos por todo el Imperio. Una visita de los lugares de Villar Tesoro, Azanaque-Castillejo y La Catria durante el pasado mes de diciembre de 2015 nos permite dar a conocer nuevos materiales."
Palabras Clave

Villar del Tesoro; Azanaque-Castillejo; La Catria; Dressel 20; Epigrafía anfórica; economía romana; distribución alimentaria.

\section{RÉSUMÉ}

Ce travail est dédié à l'étude des relations économiquesetsocialesentrelesdifférentes provinces de l'Empire Romain. Afin de connaître le rôle joué par la province Baetica dans l'administration romaine, et les politiques économiques mises en place, est nécessaire une meilleure compréhension de la production de denrées alimentaires dans la région. Pour ce faire, la découverte de conteneurs amphores fait le long du fleuve Guadalquivir et fleuve Genil, nous permet d'approfondir la production et l'exportation de la nourriture dans tout l'empire. Une visite des sites de Villar Trésor, Azanaque-Castillejo et Le Catria pendant le dernière mois de décembre de 2015 , nous permet de montrer des nouveaux matériaux céramiques avec inscriptions. Onze sont des nouvelles inscriptions, neuf des timbres sur amphores type Dressel 20 et deux sont des graphites restants. Un des graffiti est sur une Dressel 20 et l'autre sur une bassine.

\section{Mots-Clés}

Villar Tesoro; Azanaque-Castillejo; La Catria; Dressel 20; épigraphie amphorique; éoconomie romaine; distribution alimentaire.

\footnotetext{
"El presente trabajo se ha beneficiado de los recursos aportados por el proyecto de investigación Agència de Gestió d'Ajuts Universitaris i de Recerca. Generalitat de Catalunya, Centro para el Estudio de la Interdependencia Provincial en la Antigüedad Clásica (CEIPAC) (2014 SGR 218), y de la European Research Council, Economic and Political Networks (EPNet Project) (ERC-2013-ADG-340828). Ambos dirigidos por José Remesal Rodríguez.
}

jperezg@ub.edu 


\section{INTRODUCCIÓN.}

Una visita a los lugares de Villar Tesoro, Azanaque-Castillejo y La Catria durante el pasado mes de diciembre de 2015 nos permite presentar nuevos materiales epigráficos sobre cerámica. El total de inscripciones asciende a once, siendo nueve de ellas marcas impresas sobre ánforas olearias de la Bética, tipo Dressel 20, y las dos restantes, grafitos. Uno de los grafitos esta sobre el tipo de ánfora mencionado y el otro sobre un lebrillo.

Los tres lugares visitados pertenecen al territorio de Hispalis, lo que fue el conventus hispalensis (figura 1). Se integran en el vasto y complejo sistema de alfarerías productoras de ánforas Dressel 20, diseminadas a lo largo de los diferentes tramos navegables de las orillas del Guadalquivir y el Genil, en el triángulo formado por las colonias de Hispalis (Sevilla), Astigi (Écija) y Corduba (Córdoba). Así, Azanaque-Castillejo y La Catria se encuentran a orillas del río Guadalquivir, y el Villar Tesoro en los márgenes del río Corbones, en un lugar próximo a la unión de este río con el Guadalquivir. Los tres centros se sitúan en el valle del Bajo Guadalquivir, muy próximos entre sí, ubicados en el territorio de Hispalis (Corbones-Arva-Lora del Río) (Berni, 2008). A día de hoy son cerca de noventa las alfarerías conocidas a lo largo de todo el Valle del Guadalquivir.

BREVE HISTORIA DE LAS EXPLORACIONES EN LA BÉTICA

Las exploraciones del suelo andaluz se iniciaron por George Bonsor y Clark Maxwell durante la última década del s. XIX. A continuación, tras setenta años sin trabajos sistemáticos, en la década de los años setenta se ocuparon del tema M. Ponsich, J. Remesal Rodríguez y G. Chic García. Recientemente destacan los trabajos realizados por los hermanos Barea Bautista, J. Solís Siles y J. Moros Díaz sobre la Figlina Scalensia, y las excavaciones en el taller de Las Delicias bajo la dirección de E. García Vargas y S. Mauné (Mauné et al., 2014), quienes han continuado las excavaciones en el lugar iniciadas en 1997 por P. Sáez Fernández. Al margen de los estos trabajos de campo, posteriormente publicados, cabe destacar el trabajo de P. Berni Millet, hito fundamental para estudiar los sellos sobre ánforas, quien bajo una óptica global analiza todos los sellos conocidos a partir de su lugar de producción. Se trata para muchos investigadores como el punto de partida para el estudio de la epigrafía anfórica en general, y se constituirse como un referente a la hora de estudiar los complejos sistemas epigráficos que forman estas marcas y su significado.

En este sentido, el Centro para el Estudio de la Interdependencia Provincial en la Antigüedad Clásica (Ceipac), dirigido por José Remesal Rodríguez, fundado en 1989, resulta sin duda un paso obligado para aquellos interesados en conocer las inscripciones sobre el intrumentum domesticum, en particular, las ánforas. La base de datos caligráfica del Ceipac, con cerca de 41.000 registros, proporciona información, sobre todo de ánforas, especialmente, de sellos, inscripciones pintadas (tituli picti) y grafitos.

De las ánforas olearias producidas en Hispania las mejor conocidas son las del tipo definido como Dressel 20. Se trata de un tipo de ánfora con un área de producción bastante conocido, con un alto índice de sellado, y con una gran difusión en la parte occidental del Imperio romano. Por ofrecer algunos datos, a día de hoy en la base de datos del Ceipac son cerca de 23.500 las marcas de ánfora inscritas del tipo Dressel 20 conocidas en todo el Imperio romano, de las cuales alrededor de 3.800 han sido hallados en la provincia productora de la Baetica.

\section{Villar del TESORO}

La alfarería conocida como Villar Tesoro se estableció nada más remontar la orilla derecha del río Corbones. Numerosos son los restos arqueológicos que se pueden ver a lo largo del vasto campo labrado, en los que sobresalen fallos de horno de ladrillos, tejas y ánforas Dressel 20 (Ponsich, 1974, 185, nº 147; Berni Millet, 2008, 262). La marca recogida en este lugar tan sólo conserva la última letra dentro de la cartela, [---]C $\left(n^{0} 6\right)$. Creemos que se trataría de una marca FALBLIC, recogida en quince ejemplares por Ponsich (Ponsich, 1974: 974, nº 125; Ceipac, $13635,13640-13653)$ y una por Bonsor en el mismo lugar (Bonsor, 1901, 848; Ceipac, 12794). Fue producida durante el s.III d.C. de forma abundante, y cuya lectura desarrolla Berni Millet como (ex) $f$ (iglinis) Alb( ) Lic( ) (Berni Millet, 2008, 263-264, tabla 33). Se trata del único ejemplar conocido en la producción de la alfarería que finalice con la letra que presentamos aquí. Además, justo entre ésta y el final de la cartela existe un espacio que quizás pudiese haber albergado una pequeña hoja de hiedra, tan común en esta serie. 


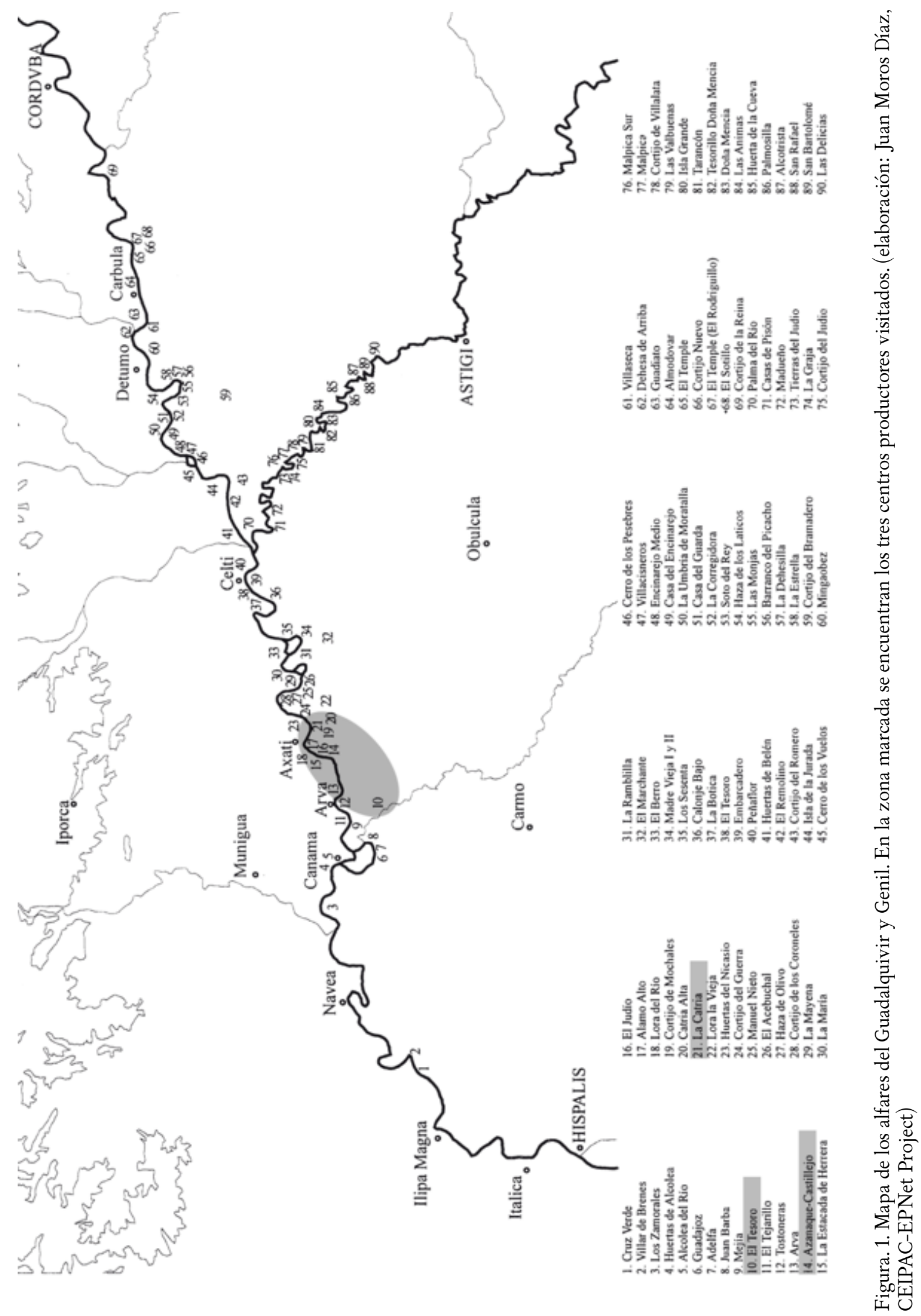




\section{AZANAQUe}

Recorriendo la margen izquierda del Guadalquivir, entre los arroyos de Azanaque y El Judío, destaca el consorcio de instalaciones industriales de Castillo de Azanaque, Azanaque-Castillejo y el Judío (Berni Millet, 2008, 305-306). Sus alfarerías ocuparon un amplio territorio, cuya actividad productiva debió ser semejante a las de otros centros productores de ánforas Dressel 20, como Arva o La Catria. Su actividad se conoce desde época de los Antoninos, intensificándose a inicios del siglo tercero y alargando su producción olearia hasta el Bajo Imperio, como constata Berni Millet al comentar el hallazgo personal de dos bocas Dressel 23 en una de las visitas al lugar (Remesal Rodríguez, 1983, 115131; Romo Salas y Vargas Jiménez, 2001,405-418; Berni Millet, 2008, 306, fig. 144).

Al lugar que Ponsich denominó como Azanaque-Castillejo (Ponsich, 1974, 193, nº 145-146, figs. 80-82; Berni Millet, 2008, 306), pertenecen las marcas CAPF y GAF recogidas en el posterior corpus (n⿳⺈ 1 y 2). La primera de las marcas, C. $A p($ ) $F($ ), con la $\mathrm{A}$ y la $\mathrm{P}$ unidas, aparece como es común en la serie con interpunciones. La marca es conocida en Azanque-Castillejo en cinco ejemplares por Ponsich (Ponsich, 1974, 145-146), y una por Bonsor (Bonsor, 1901, 155). Otro ejemplar fue hallado en el vecino centro productor de El Judío (Ponsich, 1974, nº 149; Ceipac 13275), mientras que se conoce un hallazgo residual y seguramente fortuito en el centro de Las Sesenta de en Brenes (Ponsich, 1974, no 132; Ceipac 12946). Se data en la época de Marco Antonio y Cómodo por sus hallazgos en el Monte Testaccio (CIL XV 2705; Berni, 2008, 310-312, tabla. 54). La segunda marca, GAF, G. A (p...) F ( ), es bien conocida en este lugar por los hallazgos de Bonsor (Bonsor, 1901, 161; Ceipac 12952) y Ponsich (Ponsich, 1974, $\mathrm{n}^{\mathrm{o}}$ 145-146; Ceipac 13432, 13683-13684, 1368813696 y 13731). También hallada en el Monte Testaccio, puede datarse próxima a mediados del siglo segundo, siendo quizás, como propone Berni Millet anterior a la otra marca mencionada, probablemente emparentadas (Berni Millet, 2008, 310, tabla 54). Sabemos que todos los ejemplares de la serie GAF se imprimieron con cartela ansata, por eso creemos que el presente ejemplar, afectado por el deterioro, no conserva una cartela típica de la marca.

\section{La CATRIA}

Por último, el resto de epígrafes recogidos son originarios de La Catria, considerada por Remesal como el mayor centro productor de todo el valle del Guadalquivir, con cerca de 20 hectáreas, junto a la margen izquierda del Guadalquivir, a unos $2 \mathrm{~km}$. de la actual Lora del Río (Remesal Rodríguez, 19771978, 87-142; Berni Millet, 2008, 318-319). Como demostró Remesal en las prospecciones realizadas en los años setenta con el fin de recopilar el mayor número de sellos, el estudio de unas seiscientas marcas permitió probar el gran número de nombres, variantes y formas producidas en este centro. Este hecho demostró que La Catria fue el mayor centro productor de aceite para la Annona en el occidente romano.

Además, Remesal ya destacó la distribución del aceite de la región de La Catria a campamentos militares romanos ubicados en el limes, como en Germania (Remesal Rodríguez, 1986, 50 y ss.) y Britannia (Carreras Monfort y Funari, 1998; Ayllón Martín y Pérez González, 2014, 759-763). Los datos conocidos muestran un amplio porcentaje en las importaciones olearias en las provincias fronterizas bajo dominio romano y en la capital del Imperio.

También en los trabajos de Pons Pujol se muestra el alto número de ánforas de La Catria en la Tingitana, quién comenta que pese al gran número de marcas encontradas los sellos hallados en los campamentos mauritanos son pocos y fragmentados, y quizás, a parte de tratarse de un producto annonario para las tropas allí destinadas, también pudo llegar por medio del comercio privado como complemento puntual para la demanda urbana (Pons Pujol, 2009). En esta línea, recientemente E. Garrote Sayó ha depositado su tesis doctoral sobre la distribución del aceite bético contenido en ánforas Dressel 20 a la Gallia Narbonensis, y en este trabajo comenta que también en esta provincia destaca de nuevo La Catria como el centro productor de ánforas de aceite de mayor representatividad (Garrote Sayó, 2016).

Recientemente, en el marco del proyecto EPNet (Production and Distribution of Food during the Roman Empire: Economic and Political Dynamics), un proyecto Advanced Grant concedido por la ERC (European Research Council) a J. Remesal (Remesal Rodríguez et al., 2015, 455-464), uno de los estudios iniciales ha tenido como objeto el análisis de la distribución de los materiales producidos en La Catria. El encargado de dicho estudio, X. Rubio-Campillo (BSC), me ha permitido presentar aquí los resultados visuales de los análisis realizados gracias a los datos contenidos en la base de datos del Ceipac. 
Primero, destaca una frecuencia muy alta de lugares donde se han encontrado ánforas Dressel 20, con una ratio que va de un ejemplar a cuatro ejemplares, contabilizando más de ciento veinte lugares de hallazgo diferentes en esta frecuencia. Este hecho indica la alta dispersión de las marcas de La Catria por todo el territorio romano. Por el contrario, son pocos los lugares donde el número de ejemplares supera el centenar, y entre ellos destaca Roma y alrededores con más de quinientos ejemplares diversos, muchos de ellos procedentes de las excavaciones del Monte Testaccio. En esta línea destacan ciertos lugares con una frecuencia de distribución por encima de la veintena de ejemplares hallados, como Lyon, Mainz, Londres, etc. Por último, en un último gráfico se destaca la distribución de las marcas a lo largo de los tres primeros siglos, con un alto índice productivo y distributivo desde finales del siglo primero hasta finales del siglo tercero (figura 2).
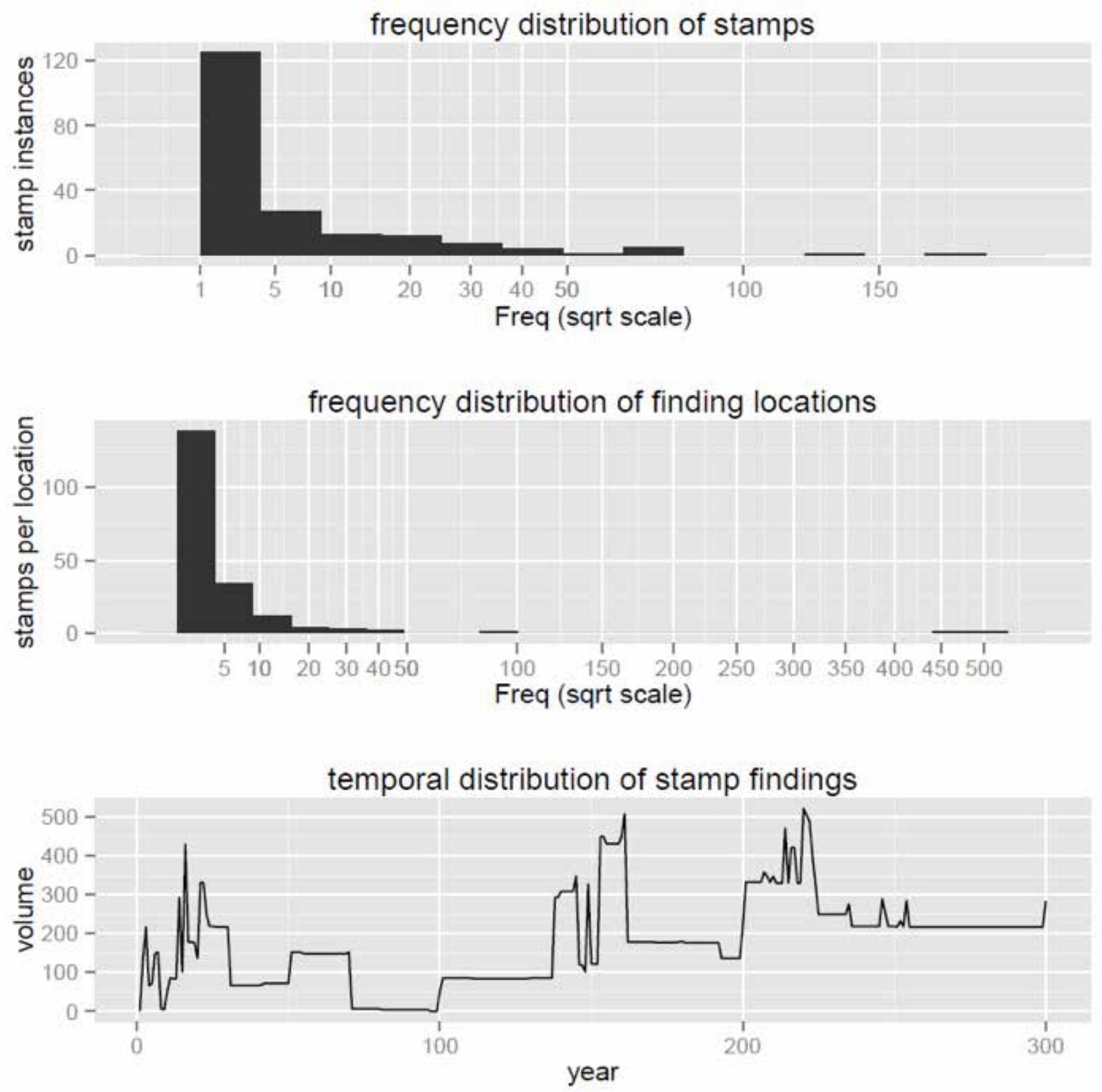

Figura 2. Arriba, gráfico de la frecuencia de distribución de los sellos sobre ánforas Dressel 20 perteneciente a La Catria. En el centro, gráfico de la frecuencia de distribución de los sellos en los lugares de hallazgo. Por último, gráfico lineal de la distribución de las marcas de La Catria. Elaboración: Xavier Rubio Campillo (BSC-EPNet Project). 


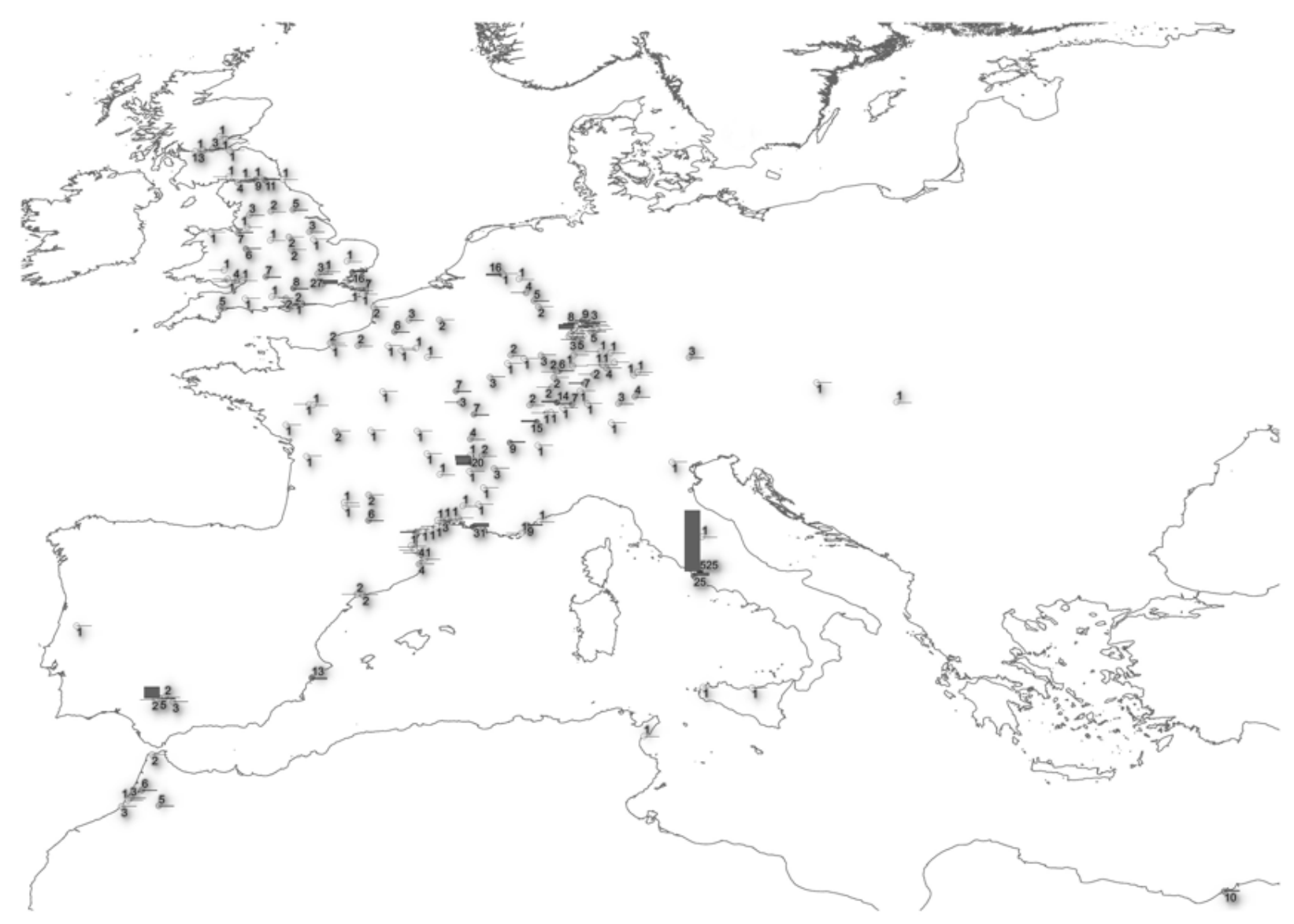

Figura 3: Mapa de los lugares donde se han encontrado sellos pertenecientes a La Catria, así como el número de ejemplares hallados.

Elaboración: Xavier Rubio Campillo (BSC-EPNet Project).

Una visualización de todos estos datos sobre un mapa viene a confirmar la teoría de Remesal, por la cual La Catria sería del mayor centro productor de aceite bético de todo el Valle del Guadalquivir, así como el de mayor distribución (figura 3).

En lo que se refiere a los sellos halados en La Catria que ahora se presentan, encontramos por dos veces la marca MIM ( $\left.n^{\mathbf{0}} 4\right)$, fragmentada en ambas ocasiones, pero que por el tipo de letra, su posición en el ánfora y su lugar de producción creemos que podemos asociarlo a esta serie. El desarrollo propuesto por Berni Millet para el sello, $M$. I( ) $M$ (opsi?), resulta como él bien indica todavía dudoso (Berni Millet, 2008, 324, tabla 57). La presente variante triliteral, además de encontrarse en La Catria por Remesal en diecisiete ocasiones (Remesal Rodríguez, 1977-1978, no 33; Ceipac 6821-6835), también se ha encontrado en las alfarerías de Las Ánimas y de Isla del Castillo, en el río Genil, por
Ponsich (Ponsich, 1991, 15-29, nº 44, Isla del Castillo; Ceipac 10883-10885) y Chic García (Chic García, 1985, 39, Las Ánimas; Ceipac 12370-12373, 12513). Haciendo coincidir las siglas del sello MIMOPSI, propio del próximo Cortijo de Los Guerras, con el personaje del siglo I d.C., M. I ( ) Mopsus, Berni Millet le otorga esa cronología (Berni Millet, 2008: 324 , tabla 57), que ha sido objeto de debate por Tchernia y Remesal (Tchernia, 1964, 419; Remesal Rodríguez, 1977-1978, 118).

Como no podría ser de otra forma, hemos encontrado dos marcas de la serie más importante de $\mathrm{La}$ Catria, SNR (nº 5), S(exti) Anni R(ufi, -ufini). De esta variante Remesal halló cuarenta y siete ejemplares, a los que hay que sumar los dos ejemplares recogidos por Chic García y uno más de Ponsich (Ponsich, 1979, no 75; Ceipac 10883-10885; Remesal Rodríguez, 1977-1978, nº 46; Ceipac 6903-6957; Chic García, 1985, 66; Ceipac, 12509 y12511). Se conoce 
una producción de mediados de siglo segundo y se han encontrado dos ánforas en el Monte Testaccio con cronologías absolutas de los años 149 y 154 gracias a los tituli picti, uno de ellos asociado al control de Astigi (CIL XV 3045b+4527 y 4294).

A continuación podemos destacar por primera vez la atribución de la marca conocida QARP $\left(\mathrm{n}^{\mathrm{o}}\right.$ 3), $Q$ ( ) $A$ ( ) $R$ ( ) P(ortus?), a La Catria, desconocida hasta el momento. Ya Berni Millet hipotetizó sobre la posibilidad de que realmente la marca fuese originaria de La Catria, así como los sellos QAR o PQAR por su indicación de portus (Berni Millet, 2008, 360, tabla 80). Estas marcas han sido fechadas a mediados del siglo II d.C. En esta línea tan sólo se conocía esta marca fuera de su lugar de producción en la Bética, siete ejemplares hallados en el Monte Testaccio (CIL XV 2662c; Ceipac 1209-1213; Remesal Rodríguez, 1999, nº 6a; Ceipac 17249; Carre et al., 1995: $\mathrm{n}^{\circ}$ 359; Ceipac 26408), y uno en Cavaliere (Pardigon, 3. Francia).

Un último ejemplar de La Catria, de dudosa lectura, ha sido propuesto dentro de la serie $M($ ) $M($ ) $R()\left(\mathrm{n}^{\mathrm{o}} 7\right)$, que pese a no conocerse en La Catria, podemos relacionar sin problemas con los sellos MMRO, MMRP, MMRCI o MMRPI, bien documentados en este centro productor (Berni Millet, 2008, n 44; Ceipac 6886-6892). Desde aquí quiero agradecer a mi compañero el Juan Moros Díaz su ayuda en la lectura del ejemplar, por la posibilidad de compararlo con las marcas inéditas en las que está trabajando para su próxima publicación y que no hacen otra cosa que reafirmar la lectura del ejemplar al comparar su tipografía.

Aparte de los sellos encontrados, destacamos el hallazgo de dos grafitos ante cocturam, uno de nuevo sobre ánfora Dressel 20 ( $\left.n^{\circ} 8\right)$ y un segundo sobre un lebrillo ( $\left.n^{\circ} 9\right)$. Pese a encontrarse fragmentado, el primero podemos atribuirlo como el numeral CX, tan común en estas ánforas sobre todo in ventre o in pede (Remesal Rodríguez et al., 2010b, 259-260, $\mathrm{n}^{\mathrm{o}} 481$ ), representado mediante una cruz inscrita dentro de un círculo. El segundo ejemplar, en la cara interna de un lebrillo, ofrece una lectura dudosa debido a que sólo se conservan algunos trazos rectilíneos paralelos entre sí, que quizás pudiesen ser parte de un grafito nominal o numeral.

Generalidades, Organización, Abreviaturas y ESTRUCTURA DEL CORPUS.

Como en trabajos anteriores relacionados con el grupo de investigación Ceipac, hemos seguido el sistema de Dressel ordenando los sellos por la letra considerada como inicial del nomen, lo que puede provocar, como se ha comentado en alguna que otra ocasión, cierto margen de error. Esta división permite agrupar los sellos por familias (Remesal Rodríguez, 1977-1978, 87-143), y dentro de los mismos grupos de nomina se ha ordenado por cognomen y praenomen. Dentro del cada nomen, se ha ordenado primero por los indicados por una sola letra, a continuación por aquellos significados con más de una letra, y finalmente por aquellos cuyo nomen esta explícitamente desarrollado. Tras ellos se mencionan los sellos que en nuestra opinión carecen de praenomen, seguidos de los que contienen cognomen, cuya letra inicial coincide con la letra nominal relativa. Aquí hemos sumando los nombres de figlinae. Al final del presente corpus se han sumado aquellos ejemplares fragmentados, ilegibles o de dudosa lectura.

Siguiendo a J. Remesal, consideramos variantes diversas aquellas que se diferencian dentro de un mismo grupo por el tamaño del sello, su puntuación, la forma o dirección de las letras, etc. Los diferentes epígrafes han sido reproducidos fotográficamente siempre a escala 1:1, y en relación con esto quiero agradecer los sabios consejos de mi compañero el J. Moros Díaz para una correcta edición de los mismos, quién ha propuesto un nuevo método de publicación de la epigrafía anfórica mediante silicona (Barea et al., 2008, 167-180).

El relieve de los sellos aquí presentados son en positivo, tan sólo los grafitos son incisos (Litteris cavis). También destacamos que en la totalidad de los sellos aquí presentados se han localizado en el asa (in ansa), como es común en la mayoría de sellos impresos sobre ánforas olearias producidas en la Baetica.

\section{EsTRUCTURA DE LAS FICHAS DEL CORPUS}

№ (de inventario): es el número de catálogo asignado para el presente trabajo.

Datación: Ofrecemos como dataciones de los epígrafes, concretamente de los sellos, dos criterios: "contextual" cuando otro ejemplar de la misma variante ha sido hallado en otro lugar cuyos estratos arqueológicos permiten datarlo por su contexto arqueológico. Un ejemplo de ello son los materiales excavados en el Monte Testaccio de Roma, donde a los sellos se les ha podido atribuir una datación por el estrato datado gracias a la presencia de tituli picti delta con fechas consulares. Aquí también se 
han incluido aquellas dataciones relacionadas con la vida de un campamento militar en el limes, lo que permite en algunos casos concretar aún más la franja productiva de una marca. El otro elemento para datar los sellos viene dado por el estudio tipológico de la cerámica, siempre que exista, mencionándose como una datación "tipológica". Un reciente estudio de P. Berni ha significado un gran avance en el conocimiento de la evolución tipológica de las ánforas Dressel 20 (Berni Millet, 2008). Se indica para ello primero el autor y el año de donde se extraen las dataciones, seguido del lugar de hallazgo de la marca. Todas las referencias bibliográficas pueden consultarse en el catálogo bibliográfico de la base de datos Ceipac.

L.H.: Lugar de Hallazgo.

L.P.: Lugar de Producción en la Bética (según Ponsich).

L.C.: Lugar de conservación.

Referencias básicas sobre cada tipo de sello(Lt.): corpora $u$ otras obras de referencia que hacen mención de la marca o a su lugar de producción. El CIL XII o XV es referido como CIL.

L.: Lectura propuesta para el sello.

Comentario: diversos comentarios sobre la marca.

Posición: en el caso de los grafitos se indica su posición.

№ 1

GAF

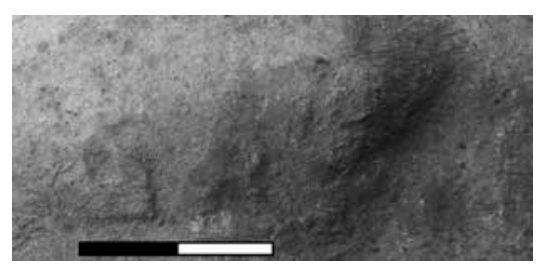

Datación: de mediados del s. II d.C. hasta el primer cuarto del siglo III d.C. Blázquez y Remesal (2001); 220-224 d.C. (contextual); CIL XV 2640; Monte Testaccio, 145-146, 149-150, 153-154, 160161 d.C. (contextual); Remesal Rodríguez, 1997, Mainz, finales s. II-s. III d.C. (Henkelform); Carreras y Funari, 1998, Loughor, 75-150 d.C. (contextual).

L.H.: Azanaque-Castillejo.

L.P.: Azanaque-Castillejo.

Lt.: CIL XV 2640c; Bonsor, 1931, 161; Ponsich, 1974, 145, y 1982, 173-204; Chic García, 1985, 77; Callender, 1965, 210b, 225; Remesal Rodríguez,
1997, 5; Carreras y Funari, 1998: 7; Blázquez y Remesal, 2001, no 4350; Ehmig, 2007, T.35.879; Silvino, 2001, 3; Étienne y Mayet, 2004, no20g. L. G ( ) A ( ) $\cdot[\mathrm{F}]$.

Comentario: La última letra resulta ilegible. Se perciben restos de una interpunción entre la $\mathrm{A}$ y lo que sería la $F$, mientras que entre la $G$ y la $A$ no observamos restos de ninguna interpunción tan común en la serie. En este sentido no se aprecian los restos de la cartela ansata que envuelve el sello característica de la variante.

№ 2

$\mathrm{CAPF}$

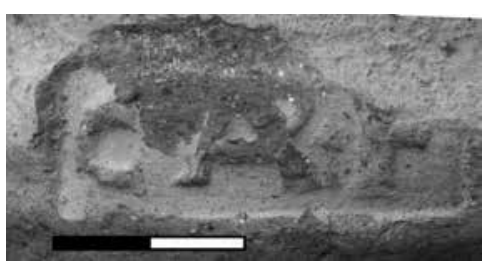

Datación: mediados y segunda mitad del s. II d.C. Romo y Vargas, 2000, APF y GAPF, segunda mitad s. II d.C.; Carreras y Funari, 1998, Benwell Hill, 142-165 d.C. (contextual.); Étienne y Mayet, 2004, s. II-III d.C. (tipológica); CIL XV (2705): Monte Testaccio: 179-180 d.C. (contextual); Meylan-Krause, 1995, Avenches: 150-250 d.C.

L.H.: Azanaque-Castillejo.

L.P.: Azanaque-Castillejo.

Lt.: Bonsor, 1931 155; CIL XV 2705; Callender, 1965, 246; Ponsich, 1974, 132; y 1982, 173-204; Carreras y Funari, 1998, 66; Lyding Will, 1983, 13; Remesal Rodríguez, 1997, 43; y 1989, 1; Remesal y Schallmayer, 1988, 4; Ehmig, 2007, T.89.469; Romo y Vargas, 2001, 413; Étienne y Mayet, 2004, 137; Rovira Guardiola, 2004, nº6.

L. C ( ) AP ( ) $\cdot \mathrm{F}()$.

Comentario: Nexo entre la A y la $\mathrm{P}$, seguida de una interpunción antes de la $\mathrm{F}$.

No 3

QARP

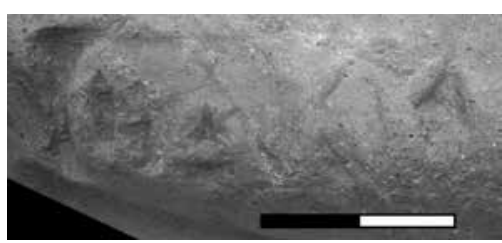


Datación: mediados del s. II d.C. CIL XV 2662c; Monte Testaccio, 145-146, 149-150, 153-154, 160161 d.C.; Blázquez y Remesal, 1999, 153-161 d.C. (contextual).

L.H.: La Catria.

L.P.: La Catria.

Lt.: CIL XV 2662; Testaccio I (1999), 6a; Callender ,1965, 1424; Carreras y Funari,1998, 67; Étienne y Mayet, 2004, 68; Brun et al., 1988, 55, $\mathrm{n}^{\mathrm{o}}$ 158, éch.1:3; Carre et al., 1995, $\mathrm{n}^{\mathrm{o}} 359$.

L. Q ( ) AR ( ) P( )

Comentario: hasta la fecha la marca es la primera de esta variante hallada en la Baetica. Del mismo ejemplar se han encontrado en el Monte Testaccio10 sellos, otro en Cavaliere (Pardigon 3) y un último en Mumrills (Falkirk).

\section{№ 4}

MIM

a1. Datación: s.I d.C.-II d.C. Jacques, 1991, Arras, s.I d.C.; Spitzlberger, 1978, Bregenz, 30-80/90 d.C.; Schimmer, 2009, Cambodunum (Kempten), 30-70 d.C.; Carreras y Funari, 1998, London, 40-100 y 6996 d.C.; Paunier, 1981, Genéve ca. s. I d.C.;Bertrand, 1992, Saint-Just (Lyon), 50-100 d.C.; Hassini, 2009, Thamusida, 40-70 d.C.; Corault, 2013, Thononles-Bains, 90-120 d.C.; Leblanc, 1994, Saint-Romain-en-Gal, ca. 30/50-70/100 d.C.;Marlière, 2003, Vindolanda, 160-180 d.C.; CIL XV, 2933, Monte Testaccio, 145, 147, 149, 150, 153-154, 160-161 d.C.; Rodríguez Almeida, 1977, Monte Testaccio, 98192 d.C.

L.H.: La Catria.

L.P.: La Catria.

Lt.: Chic García, 1985, 344-345; Ponsich, 1991, 44;, 1977-78: n⿳0 33; y 1986, nº138 ; Remesal Rodríguez, 1997, no 165; Leblanc, 1994, 27-28; Ehmig, 2003, 102,7; Ehmig, 2007, T.62.229; Carreras y Funari, 1998, n² 244; CIL XII, 5683.136d; CIL XV, 2933; Corault, 2013, 183; Hassini, 2009, 31; Bertrand, 1992, 10; Marlière, 2003, 27; Étienne y Mayet, 2004, nº635; Lyding Will, 1983, 38; Rovira Guardiola, 2004, n⿳028.

$$
\text { L. } \mathrm{M}() \mathrm{I}()[\mathrm{M}()]
$$

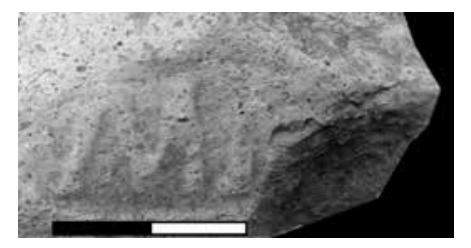

a2. L. $[\mathrm{M}($ ) $\mathrm{I}(\mathrm{)})] \mathrm{M}($ )

Comentario: el sello aparece en zona superior del asa, marcado en horizontal.

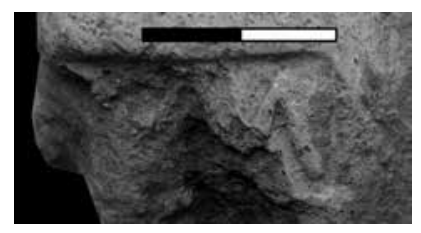

№ 5

SNR

a1. Datación: s. II d.C. CIL XV (3045); Monte Testaccio, 145-146 147, 149-150, 153-154, 160, 161 d.C. (contextual); Blázquez y Remesal, 1999, Monte Testaccio, 153-161 d.C. (contextual); Carreras y Funari, 1998, Bar Hill, Bearsden, Cadder (Bishopbriggs) y Corbridge, 142-165 d.C. (contextual); Jacques, 1991, Boulogne, 140-170 d.C.; Laubenheimer y Marlière, 2010, Amiens, 110-180 d.C.

L.H.: La Catria.

L.P.: La Catria. Tan sólo un ejemplar fue hallado en el Cortijo del Guerra, en La Catria conocemos 49 ejemplares sin contar los catalogados aquí.

Lt.: CIL XV 3045; Chic García, 1985, 593, 595; Callender, 1965, no 47; Remesal Rodríguez, 197778, nº 46; Remesal Rodríguez, 1997, nº 3; Blázquez y Remesal, 1999, n 23; Carreras y Funari, 1998, nº 339; Ehmig, 2007, T.28.1466, T.36.822; Étienne y Mayet, 2004, $\mathrm{n}^{\mathrm{O}} 850 \mathrm{k}-\mathrm{n}$; Laubenheimer y Marlière, 2010, no 147 .

$$
\text { L. S ( ) N( ) R( ) }
$$

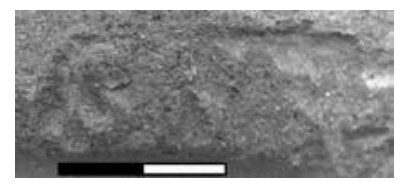

a2. L. S( ) $\mathrm{N}($ ) $[\mathrm{R}()]$

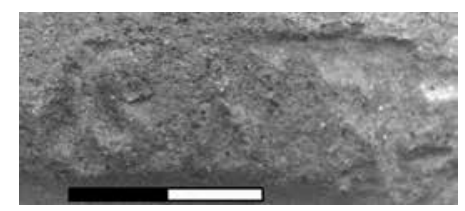

№ 6

[---]C

LH: Villar Tesoro.

L.P.: Villar Tesoro.

L. [---] C. 
Comentario: Quizás se trate del sello FALBLIC perteneciente al lugar de Villar Tesoro, tratándose hasta la fecha de la única variante finalizada con la letra C. Es posible que entre la $\mathrm{C}$ y el final de la cartela hubiese una pequeña hoja de hiedra, que por el deterioro de la pieza no se ha conservado. Véase: Bonsor, 1931, 335; Ponsich, 1974, 125; Ponsich, 1982, 173-204; Étienne y Mayet, 2004, nº113; Ceipac 13126, 13635, 13640-13653.

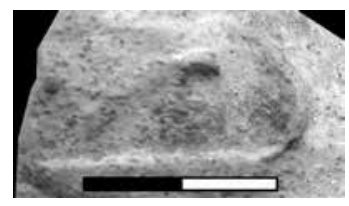

$\mathrm{N}^{\mathrm{o}} 7$

MMR

Datación: producción flavio-trajanea (según comentarios de P.Berni Millet)

L.H.: La Catria.

L.P.: La Catria.

L. [---] MM·R

Comentario: En el lugar de La Catria se han documentado diversas marcas con doble $\mathrm{M}$, al estilo de esta marca, con letras muy finas y estilizadas que incluso parecen unir en los trazos finales. Quizás pueda atribuirse al conjunto de marcas de los sellos publicados por Remesal Rodríguez, 197778, no 44, MMR..., MMRCI, MMRO, MMRP, etc. (Ceipac, no 6892, 6886-6891).

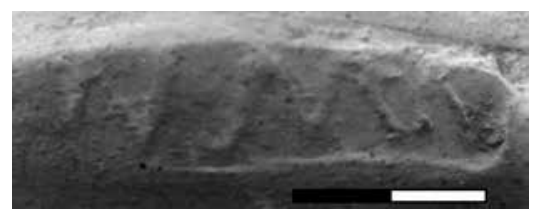

\section{$\mathrm{N}^{\mathrm{o}} 8$}

Grafito numeral sobre ánfora Dressel 20 in ventre - in pede.

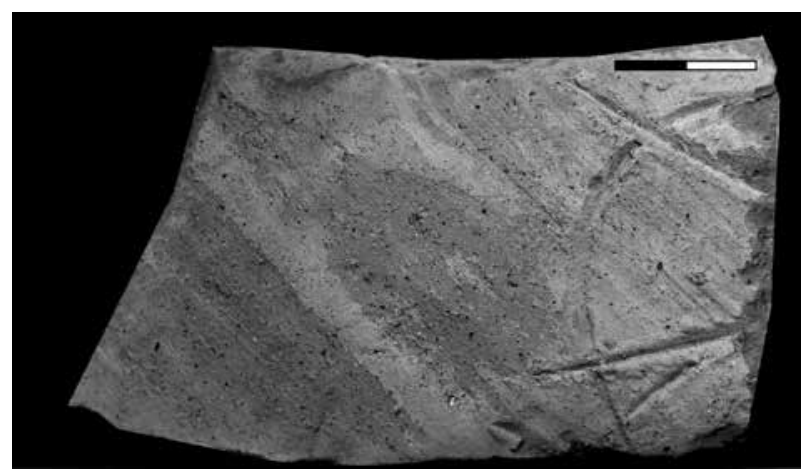

L.H.: Superficial. La Catria.

Posición: in ventre - in pede.

Lectura: CX.

Comentario: restos de círculo partido por dos trazos rectilíneos que se cruzan entre sí definiendo una cruz, más o menos centrada y que aquí no conservamos entera.

№ 9

Grafito interno en lebrillo.

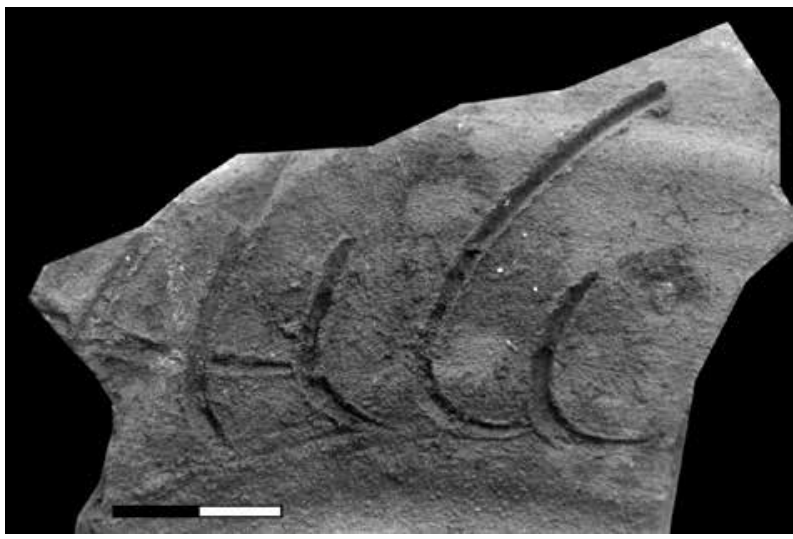

L.H.: Superficial. La Catria.

Posición: in ventre - in pede.

Comentario: restos de trazos rectilíneos paralelos entre sí en la cara interna de lo que parece un lebrillo. Quizás podría tratarse del nombre de artesano fabricante o de un numeral. Resulta de difícil lectura.

\section{ADDENDA}

Al margen del presente trabajo de los materiales encontrados en la Bética, deseamos incluir en última instancia un ejemplar hallado en uno de los patios traseros de los edificios habitables anexos a las faldas del Monte Testaccio en Roma, presentes desde la construcción del actual barrio Testaccio, acompañado del complejo cárnico del Mattatoio (Ramieri, 2007, 395), el sello PNN ( $\left.\mathrm{n}^{\mathrm{O}} 10\right)$. El ejemplar fue descubierto en la parte inferior de una fuente realizada con cerámica, en la vertiente occidental del Testaccio, en la littera $\mathrm{H}$ descrita por $\mathrm{H}$. Dressel, donde aparecieron materiales severianos. Desconocemos si realmente la pieza pertenece a esta zona del monte o si bien es una inferencia descontextualizada. Pese a ello la datación se corresponde con la de la marca. Creemos también interesante presentar el sello para posibles estudios tipográficos de una de las marcas más frecuentes halladas en el Testaccio, el sello PNN. 
El ejemplar procede de la zona de Arva/Canama, y corresponde a uno de los personajes más representativos en la producción olearia de la Béti$\mathrm{ca}$, abundante en todos los hallazgos del occidente romano, del que se han contabilizado con éste según la base de datos Ceipac 356 ejemplares. Se deduce que quizás el personaje bajo esta marca se viese beneficiado de las confiscaciones y ventas de las propiedades llevadas a cabo en el territorio por los Severos. Su producción se extendió entre Arva y El Tejarillo (Remesal Rodríguez, 2010b, 240).

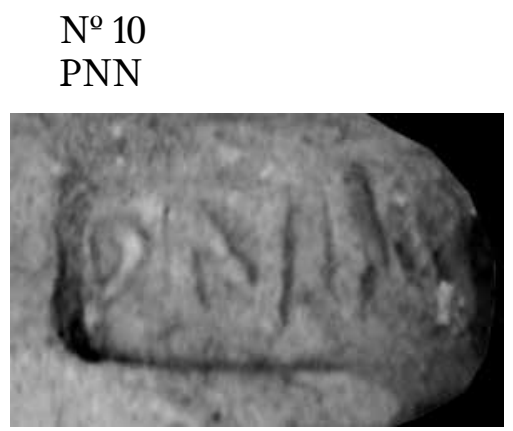

Datación.: primer cuarto del s. III d.C. 207-208, 210, 214, 216-217, 220- 224 d.C., 201-300 d.C. CIL XV Occ. III

1/2-3/4 in situ (s. III d.C.). Blázquez, Remesal y Rodríguez, 1994, ca. 220-224 y supra. Testaccio. II (2001), ca. 220- 224 infra y supr. (3187). 207-208, 210, 214, 216-217, 223 d.C.

L.H.: Monte Testaccio. En una fuente de un patio trasero de un edificio habitable. Littera $\mathrm{H}$.

L.P.: Arva, El Tejarillo, Alcolea del Río, Tostoneras, Sevilla.

Lt.: Bonsor, 1931, n 217-227; CIL XV 3041; Ponsich, 1974, no 54; Carreras y Funari 1998, 338; Blázquez et al., 1994, n⿳0 287; Blázquez y Remesal, 2001, n 463 y 485; Blázquez y Remesal, 2003, n ${ }^{\circ}$ 898; Blázquez y Remesal, 2010c, nº 384; Étienne y Mayet, 2004, no 836am.

$$
\text { L. } \mathrm{P}() \mathrm{N}() \mathrm{N}(\text { ) }
$$

\section{Agradecimientos}

Agradezco las correcciones desinteresadas realizadas por Piero Berni Millet y Juan Moros Díaz, y aprovecho la ocasión para recordar a nuestro maestro y mentor el Prof. José María Blázquez Martínez, quién nos abandonó recientemente. Las últimas palabras de agradecimiento se las dedico a mi esposa Jéssica.

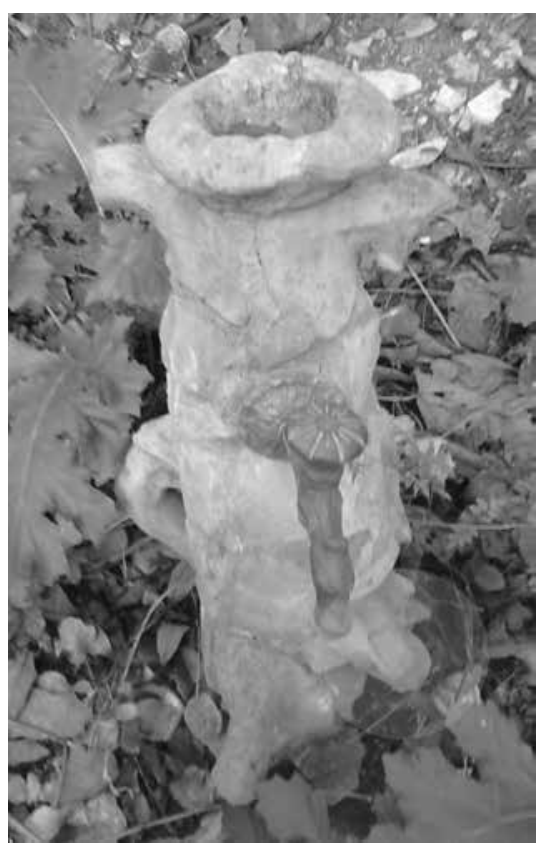


Bibliografía

Ayllón Martín, R.; Pérez González, J. (2014), “Oro líquido en los confines del Mundo Romano: la ruta del aceite bético desde las figlinae hasta el Muro de Adriano", Centro y periferia en el mundo clásico, Las vías de comunicación en Grecia y Roma: rutas e infraestructuras, XVIII Congreso Internacional de Arqueología Clásica, Mérida, 759-763.

Barea Bautista, J.S., Barea Bautista, J., Solís Siles, J., y Moros Díaz, J.M. (2008), Figlina Scalensia: Un centro productor de ánforas Dressel 20 de la Bética, Instrumenta 27, Barcelona.

Berni Millet, P. (2008), Epigrafía anfórica de la Bética. Nuevas formas de análisis, Instrumenta 29, Barcelona.

Bertrand, E. (1992): "Les amphores d'un vide sanitaire du 1er siècle à Lyon (Saint-Just)", Societé francaise d'étude de la ceramique antique en Gaule, Actes du Congrès de Tournai (L. Rivet, ed.), Marseille, 265-277.

Blázquez Martínez, J.M. y Remesal Rodríguez, J. -eds.- (1999), Estudios sobre el Monte Testaccio (Roma) I, Instrumenta 6, Barcelona.

Blázquez Martínez, J.M. y Remesal Rodríguez, J. -eds.- (2001), Estudios sobre el Monte Testaccio (Roma) II, Instrumenta 10, Barcelona.

Blázquez Martínez, J.M. y Remesal Rodríguez, J. -eds.- (2010), Estudios sobre el Monte Testaccio (Roma) $V$, Instrumenta 35, Barcelona.

Blázquez Martínez, J.M.; Remesal Rodríguez, J., Rodríguez Almeida, E. -eds.- (1994), Excavaciones Arqueológicas en el Monte Testaccio (Roma). Memoria de la Campaña de 1989, Madrid.

Bonsor, G.E. (1901): "Los pueblos antiguos del Guadalquivir y las alfarerías romanas", Revista de Archivos, Bibliotecas y Museos, 12, 837-857.

Bonsor, G.E. (1931), The Archaeological Expedition along the Guadalquivir, New York.

Brun, J. P., Congès, G., y Bewert, J. P. (1988), "La villa gallo-romaine de Pardigon 3", $A u$ tour d'Heraclea Caccabaria. Catalogue de l'exposition Archéologie de la côte des Maures.

Callender, M.H. (1965), Roman Amphorae (with an Index of Stamps), Oxford University Press, London.

Carre, M.B., Gaggadis-Robin, V., Hesnard, A., y Tchernia, A.M.B. (1995), Recueil de timbres sur amphores romaines (1987-1988), Aix en Provence.
Carreras Montfort, C. y Funari, P.P.A. (1998), Britannia y el Mediterráneo: Estudios sobre el abastecimiento de aceite bético y africano en Britannia, Instrumenta 5, Barcelona.

Chic García, G. (1985), Epigrafía anfórica de la Bética I: las marcas impresas en el barro sobre ánforas olearias (Dressel 1920 23), Universidad de Sevilla, Sevilla.

Corault, C. (2013), "La Bética en los Alpes: análisis de sellos de ánforas Dressel 20 descubiertos en Thonon-Les-Bains", Arte, Arqueología e Historia, 20, 183-188.

Ehmig, U. (2003), Der Römischen Amphoren aus Mainz, Frankfurter Archäologische Schriften, 4, Möhnesee.

Ehmig, U. (2007), Die romischen Amphoren im Umland von Mainz, Frankfurter Archäologische Schriften, 5, Reichert verlag Wiesbaden.

Étienne, R. y Mayet, F. (2004), L'huile hispanique. Corpus des timbres amphoriques sur amphores Dressel 20, De Boccard, Paris.

Garrote Sayó, E. (2016), La presència de l’oli bètic a la Gallia Narbonensis, Tesis Doctoral (depositada e inédita), Universitat de Barcelona.

Hassini, H. (2009), Nouvelles marques sur amphores au Maroc, Bulletin d'Archéologie Marocaine, 21, 234-245.

Jacques, F. (1991), L'huile de Bétique dans le nord de la France d'après les marques d'amphores Dressel 20, Revue du Nord, 73/292, 195-223.

Laubenheimer, M. y Marlière, E. (2010), Échanges et vie économique dans le Nord-Ouest des Gaules. Le témoignage des amphores du IIe siècle avant J.-C. au IVe siècle après J.-C., Institut des Sciencies et Techniques de l'Antiquité, Paris.

Leblanc, O. (1994), Étude du mobilier et chronologie. Évolution Urbaine à Saint-Romainen-Gal (J.L. Prisset, L. Brissaud, y O. Leblanc, O., Eds.), Gallia, 51, Paris.

Lyding Will, E. (1983): "Exportation of Olive Oil from Baetica to the Eastern Mediterranean", Segundo Congreso Internacional Producción y Comercio del Aceite en la Antigüedad (J.M ${ }^{\mathrm{a}}$. Blázquez Martínez y J.M. Remesal Rodríguez, eds.), Madrid, 391-440.

Marlière, E (2003), “Tonneaux et amphores à Vindolanda contribution à la connaissance de l'approvisionement des troupes stationnés sur la frontière Nord de l'Empire", Vindolanda. Excavations 2001-2002, I (A. Birley, ed.), Lon- 
don, 128-179.

Mauné, S., García, E., Bourgeon, O., Carrato, Ch., García-Dils, S., Corbel, S., Bigot, F., y Vázquez, F. (2014), "L'atelier d'amphores à huile Dr. 20 de Las Delicias à Ecija (Prov. de Séville, Espagne). Résultats de la campagne de fouille 2013 et perspectives", Actes du Congrès International de la Société Française d'Etude Céramologique Antique en Gaule (L. Rivet y S. Saulnier, dirs.), Marseille, 419-444.

Paunier, D. (1981), La Céramique Gallo-Romaine de Genève, Mémoires et documents publiés par la Société d'histoire et d'archéologie de Genève, Série 4, Tome, 9, Genève-Paris.

Pons, Ll. (2009), La Economía de la Mauretania Tingitana (s. I-III d.C.). Aceite, vino y salazones, Instrumenta 34, Barcelona.

Ponsich M. (1974), Implantation Rurale Antique sur le Bas-Guadalquivir, Laboratoire d'archéologie de la Casa de Velázquez, Madrid.

Ponsich M. (1979), Implantation Rurale Antique sur le Bas-Guadalquivir, Paris.

Ponsich M. (1982): "Marcas de ánforas de aceite de la ribera del Betis", Archivo Español de Arqueología, 55, 173-204.

Ponsich M. - (1991), "Prospección arqueológica: metodología para la lectura de un paisaje en la antigüedad", Almoraima, 5, 15-30.

Ramieri, A.M. (2007), "Roma e il Testaccio tra Storia, Costume e Tutela”, Estudios sobre el Monte Testaccio (Roma), IV (J.Mª Blázquez Martínez y J. Remesal Rodríguez, eds.), Instrumenta 24, Barcelona, 381-400.

Remesal Rodríguez, J. (1977-1978), “La economía oleicola bética: nuevas formas de análisis", $A r$ chivo Español de Arqueología, 50-51, 87-142.

Remesal Rodríguez, J. (1986), La Annona Militaris y la Exportación de Aceite Betico a Germania, Ediciones de la Universidad Complutense, Madrid.

Remesal Rodríguez, J. (1983), “Transformaciones en la exportación del aceite bético a mediados del siglo III d.C.", Segundo Congreso Internacional Producción y Comercio del aceite en la Antigüedad (J.Mª Blázquez Martínez y J. Remesal Rodríguez, eds.), Madrid, 115-129.

Remesal Rodríguez, J. (1997), Heeresversorgung und die wirtshaftlicjen beziehungen zwischen der Baetica und Germanien-Corpus der in Deutschland gefundenen stempell auf amphoren Dr.20, Stuttgart.
Remesal Rodríguez, J. (1999): "Los sellos”, Estudios sobre el Monte Testaccio (Roma) I (J.M Blázquez Martínez y J.Remesal Rodríguez, eds.), Instrumenta 6, Barcelona, 31-48.

Remesal Rodríguez, J. (2010): "Los grafitos”, Estudios sobre el Monte Testaccio (Roma), V (J.M ${ }^{a}$ Blázquez Martínez y J. Remesal Rodríguez, eds.), Instrumenta 35, Barcelona.

Remesal Rodríguez, J., Calzada Baños, S., Rovira Guardiola, R., y Soria Rincón, J. (2010): “Los grafitos”, Estudios sobre el Monte Testaccio (Roma), V(J.M Bázquez Martínez y J. Remesal Rodríguez, eds.), Instrumenta 35, Barcelona.

Remesal Rodríguez, J., Díaz-Guilera, B., Rondelli, B., Rubio-Campillo, X., Martín-Arroyo, D.J., Mosca, A., y Rull, G. (2015), "The EPNet Project. Production and distribution of food during the Roman Empire: economics and political dynamics", Information Technologies for Epigraphy and Cultural Heritage, Editrice, 455-464.

Remesal Rodríguez, J.y Shallmayer, E. (1988), "Römische Amphoren aus Baden-Württemberg”, Fundberichte aus Baden-Württemberg, 13, 395-432.

Rodríguez Almeida, E. (1977), Bolli anforari di Monte Testaccio, Bulletino della Comissione Archeologica di Roma, 84, Roma.

Romo Salas, A. y Vargas Jiménez, J.M. (2001): Azanaque. Evidencias arqueológicas de un centro de producción anfórica, Congreso Internacional Ex Baetica Amphorae, I, Écija, 405-417.

Rovira Guardiola, R. (2004), Las Relaciones comerciales entre Hispania y las provincias orientales durante el Alto Imperio Romano, Tesis doctoral (inédita). Universitat de Barcelona.

Schimmer, F. (2009), Amphoren aus Cambodunum/Kempten. Ein Beitrag zur Handelsgeschichte der römischen Provinz Raetia, Münchner Beiträge zur Provinzialrömischen Archäologie, Reichert Verlag Wiesbaden.

Silvino, T. (2001), "Les importations d'amphores à huile de Bétique à Lyon du Ier siècle av. J.-C. Au IIIe siècle apr. J.-C.", Societé francaise d'étude de la ceramique antique en Gaule, Actes du Congrès de Lilli-Bavay, Marseille, 331-346.

Spitzlberger, G. (1978), "Die römischen Amphorenstempel von Bregenz, zum exporthandel südspaniens im 1. und 2. Jarhundert n. Chr.", Jahrbuch des Vorarlberger Landesmuseum- 
88 Nuevos ejemplares de marcas sobre ÁnForas Dressel 20 en el territorio del Conventus Hispalensis

svereins, Vorarlberger Landesmuseumsverein, Bregenz, 157-184.

Tchernia, A. (1964), "Amphores et marques d'amphores de Bétique a Pompei et à Stabies", Mélanges d'archéologie et d'histoire de l'École Française de Rome, 76 (2), 419-449. 